\title{
Technological platforms used for the screening of new antiviral drugs
}

\begin{abstract}
One of the main characteristics of viruses is their high genetic diversity that generates viral variants with mutations associated with the resistance of antiviral drugs, which leads to therapeutic failure, increases morbidity and mortality and decreases the quality and life expectancy of infected individuals. That is why the need arises to find new alternatives that accept changes in the strongest and most effective devices. Modern pharmacological designs can be made through technological platforms, in a faster, cheaper and more effective way, which offer new promising compounds that act in host-virus binding sites. In this review, we describe the pharmacological objectives and the use of technological tools for the development of new antiviral drugs.
\end{abstract}

Keywords: high throughput screening, biosensors, microfluidics, antiviral drugs, virus-host interactions
Volume 4 Issue 2 - 2018

\author{
Sandra Janneth Perdomo Lara, Diana Marcela \\ Buitrago Ramirez \\ Unit of Basic Oral Investigation-UIBO, El Bosque University, \\ Colombia
}

Correspondence: Diana Marcela Buitrago Ramirez, Unit of Basic Oral investigation-UIBO, Laboratory of Biotechnology, School of Dentistry, Universidad El Bosque, I 10121 , Bogota' DC, Colombia, Cra 9 No I3I A - 02, Tel 0057 I-6489000 ext I I58, Email buitragodianam@unbosque.edu.co

Received: February 07, 20I8 | Published: March 09, 2018
Abbreviations: WHO, world health organization; VH, virus-host, HTS, high throughput screening; PPI, protein-protein interactions; HIVp, HIV protease; CADD, computer-aided drug design

\section{Introduction}

Currently, virus infections continue to be a public health problem, mainly affecting developing countries. ${ }^{1,2}$ According to the World Health Organization (WHO), infections by viruses such as HIV and hepatitis are among the 10 leading causes of death worldwide ${ }^{3}$ which entails a greater expenditure on health by the government. The preventive measures used in viral infections are mainly based on the development and use of vaccines that can provide long-term protection, however they refer only to a limited number of viruses. ${ }^{2}$ The drugs used for the treatment of virus infections act on a specific component or enzyme in the processes of viral penetration and replication, however the great disadvantage is that viruses have the ability to mutate rapidly because their low fidelity replication process which leads to a high resistance to drugs. ${ }^{1,4}$ Currently the development of new drugs is focused on finding new therapeutic alternatives, more effective and that help to reduce the resistance of viral strains. Studies have begun to explore the possibility of developing molecules directed to viral replication processes associated with host-virus interactions, specifically proteinprotein (PPI) or protein-ligand interactions. ${ }^{4,5}$ High performance screening (HTS) as biosensors and microfluidics are tools that can be used to elucidate mechanisms of interaction of host-virus proteins through the generation of intermolecular models that allow the rapid and selective selection of antiviral pharmacological candidates in the phase initial that act specifically on the target site of interest., Below is a brief description of the development of HTS designed for the screening of molecules with antiviral potential associated with the host-virus interactions.

\section{Discussion}

The development of treatments to combat diseases caused by viral agents has led to the development of many advances in technological platforms to improve clinical trials. These advances have generated miniaturized analytical devices "biosensors" that incorporating a biological sensitive recognition element immobilized on a physicochemical transducer to measure one or more biomarkers. ${ }^{6}$ The biosensors combined with microfluidic systems technology increase sensitivity of detection of a specific analytes using optical transduction or electrochemical systems provides the ability to merge chemical and biological components into a single platform. The application of biosensors in the field of pharmaceuticals has been increasing, allowing a high-throughput screening of combinatorial libraries for the detection of new antiviral molecules. ${ }^{5,7}$ The viral infections activate a complex network of molecules and uncountable number of interactions and transformations interconnect all the system's components. In addition to these biochemical components the location of all of these interactions plays an important role in the defense of the host against viral infection. The molecular mechanisms of viral infections and the response of the host cells are widely varied. However, as protein complexes and their interaction networks into which they are organized comprise the primary functional modules of the cell, ${ }^{6}$ and the disruption of these host networks are likely to be a key strategy for manipulation by pathogens. The identification of virus-host interactomes is essential for the discovery of a network of cellular proteins essentials in the virus life cycle. These proteins can be considered as new therapeutic targets for antiviral drugs and small molecules (miRNAs, RNAi) and may include viral, structural and nonstructural proteins and evaluate specific affinities of proteinprotein, protein-carbohydrate or protein-DNA interactions. ${ }^{8}$ Thus, mapping the host-pathogen protein interactome may provide valuable insights into the biological functions of virulence factor proteins, highlight interactions critical to the pathogens' progression, 
and improve our overall understanding on the molecular basis of pathogenicity. ${ }^{9}$ Recent advances in technology provide opportunities to overcome the limitations of traditional methodologies: any new analytical platform for this purpose must (a) facilitate incorporation of human an viral proteins in a single platform, (b) resolving the behavior of individual proteins in the viral life cycle, (c) identify altered signaling pathways, (d) identify therapeutic targets with quick, practical, affordable, and reliable assays. Microfluidic platforms, miniaturized devices with micrometer-sized channels and chambers, are good candidates for all of these requirements. ${ }^{10}$ Thus, mapping the host-pathogen protein interactome may provide valuable insights into the biological functions of virulence factor proteins, highlight interactions critical to the pathogens' progression and spread, and improve our overall understanding on the molecular basis of pathogenicity.

Recent studies have shown that the use of technological tools for the identification of new antiviral drugs that act in PPI sites, has shown that these techniques allow to increase the effectiveness of the process of identification of new antiviral drugs of broad spectrum. In order to find a treatment or cure for HIV, studies have focused on using platforms that allow to find new drugs that act in the PPI sites of the host-virus, Hou et all developed an artificial ion channels biosensors for the detection of gp41 inhibitors (virus-host fusion glycoproteins), using an electrochemical transduction signal. The sensor was constructed from the domain of the hydrophobic region of gp41, which can be blocked by new molecules that act as fusion inhibitors and as it does not require the identification of the C-terminal peptide domain, which offers a promising application for extending the assay to a variety of HIV-1 viral fusion domains. ${ }^{11}$ Likewise, Bozal et al., ${ }^{12}$ using the "in silico" computational technique, has evaluated the specific points of mutations that occur in HIV proteases (HIVp), specifically in the formation of alanine dimers; main factor of resistance to currently used protease inhibitor drugs, in order to identify compounds through a computational screening that can act directly in these points and later be evaluated in vitro using an optical chip biosensor (CM5), in order to find compounds that can dissociate the dimers from HIVp monomers. ${ }^{12}$ Technological platforms have also been used in order to obtain bioactive compounds from traditional medicine; for example, Computer-aided drug design (CADD) systems are very useful for quickly and efficiently determining molecules of interest. Through these systems, a virtual record of the binding sites to viral proteins or ligands of the host cell is made, and then In vitro techniques such as biosensors, microfluidics or 3D assays selectively identify the compounds with the highest antiviral potential. ${ }^{13,14}$ These new studies have the advantage of identifying drugs with greater efficacy, safety and minimal viral resistance.

\section{Conclusion}

Host-virus interactions represent a strategy to identify new potential therapeutic targets, therefore it is important to develop a suitable technological platform that allows identifying and analyzing these interactions in a fast and efficient way, mainly those associated with protein-protein and protein-receptor. The design of a computer bioinformatics software that allows to identify new therapeutic targets; such as: viral cell binding proteins, intracellular vesicle absorption (host), viral fusion proteins, integration of viral DNA into the host cell
DNA, protein translation and post-translational signaling that can be used for the development of platforms technological as; biosensors or microfluidics that are used for the analysis of molecules with potential antiviral activity.

\section{Acknowledgements}

None.

\section{Conflict of interest}

The author declares that they have no conflict of interest.

\section{References}

1. De Chassey B, Meyniel-Schicklin L, Aublin-Gex A, et al. New horizons for antiviral drug discovery from virus-host protein interaction networks. Curr Opin Virol. 2012;2(5):606-13.

2. De Chassey B, Meyniel-Schicklin L, Vonderscher J, et al. Virushost interactomics: New insights and opportunities for antiviral drug discovery. Genome Med. 2014;6(11):1-14.

3. Bautista-Villa, Abel Félix Gustavo Mora-Ríos, Mejía-Rohenes C, et al. Ambientes saludables y prevención de enfermedades, hacia una estimación de la carga de morbilidad atribuible al medio ambiente. Organ Mund la Salud. 2015;1(2):16.

4. Ivanov AS, Gnedenko OV, Molnar AA, et al. Protein-protein interactions as new targets for drug design: virtual and experimental approaches. J Bioinform Comput Biol. 2007;5(2b):579.

5. Zang R, Li D, Tang IC, et al. Cell-Based Assays in High-Throughput Screening for Drug Discovery. Int $J$ Biotechnol Wellness Ind. 2012;1(1):31-51

6. Mehrotra P. Biosensors and their applications - A review. J Oral Biol Craniofacial Res. 2016;6(2):153-9.

7. Fraietta I, Gasparri F. The development of high-content screening (HCS) technology and its importance to drug discovery. Expert Opin Drug Discov. 2016;11(5):501-14.

8. Lazcka O, Campo FJ Del, Muñoz FX. Pathogen detection: A perspective of traditional methods and biosensors. Biosens Bioelectron. 2007;22(7):1205-17.

9. Nicod C, Banaei-Esfahani A, Collins BC. Elucidation of hostpathogen protein-protein interactions to uncover mechanisms of host cell rewiring. Curr Opin Microbiol. 2017;39:7-15.

10. Coupland P, Batchelor M, Convine N, et al. Lab on a Chip. 2010;10:531-40.

11. Hou Y, Gochin M. Artificial ion channel biosensor in human immunodeficiency virus gp41 drug sensing. Anal Chem. 2008;80(15):5924-9.

12. Bozal B, Uslu B, Özkan SA. A Review of Electroanalytical Techniques for Determination of Anti-HIV Drugs. Int J Electrochem. $2011 ; 17$ p.

13. Minunni M, Tombelli S, Mascini M, et al. An optical DNA-based biosensor for the analysis of bioactive constituents with application in drug and herbal drug screening. Talanta. 2005;65(2):578-85.

14. $\mathrm{Xu} \mathrm{X}$. New concepts and approaches for drug discovery based on traditional Chinese medicine. Drug Discov Today Technol. 2006;3(3):247-53. 